\section{JTI}

JOURNAL OF

TRAUMA AND INJURY

\title{
Exceptionally Unusual Case of a Self-Inflicted Suicidal Cut Throat Injury
}

\author{
Mezhuneituo Raleng, M.S. ${ }^{1}$, Anant Prakash Pore, D.N.B ${ }^{1}$, Temsula Alinger, M.S. ${ }^{2}$ \\ ${ }^{1}$ Department of Surgical Oncology, Tata Medical Center, Kolkata, India \\ ${ }^{2}$ Department of General Surgery, Christian Institute of Health Sciences and Research, \\ Nagaland, India
}

Received: December 16, 2019

Revised: March 8, 2020

Accepted: March 22, 2020

\section{Correspondence to}

Mezhuneituo Raleng, M.S

Department of Surgical Oncology, Tata Medical Center, 14 MAR (E-W), DH Block

(Newtown), Action Area I, Newtown Kolkata, West Bengal 700160, India

Tel: +91-879-470-7032

Fax: $+91-33-6605-7000$

E-mail:neituo@gmail.com
Here we present a 43-year-old man who was brought with a self-inflicted cut throat injury; 18 hours after the suicidal attempt. On examination a deep $12 \mathrm{~cm}$ cut at the level of the hyoid bone exposing the posterior pharyngeal wall was seen. Emergency surgery with primary repair, tracheostomy and feeding gastrostomy was done. Post-operative period was uneventful and patient recovered without any speech or swallowing abnormalities. Through this article we would like to stress that even in cases of frightening ghastly wounds, by maintaining simple surgical principles we can achieve good outcomes.

Keywords: Throat; Injuries; Neck; Suicide

\section{INTRODUCTION}

Suicidal cut throat injuries are regularly encountered by surgeons, but usually are limited to superficial wounds and hesitation cuts. It is very rare for someone to survive a major suicidal cut throat injury, and relevant management protocols and reports are therefore sparse in the literature. By reporting this highly unusual case, it is our aim to add to the pool of reports on major cut throat injuries and thereby augment the knowledge of this phenomenon among the medical community.

\section{CASE REPORT}

A 43-year-old alcoholic male patient was brought from a primary health center $250 \mathrm{~km}$ 
away, with a history of a self-inflicted suicidal neck injury, almost 18 hours after the injury was inflicted. Upon presentation, the patient was conscious and cooperative. He had a 12-cm gaping wound on the neck and he was breathing through the open wound (Fig. 1). The wound was crudely dressed with pieces of gauze and his neck was covered with a piece of cloth. There was no active bleeding, and the patient's vitals were stable, with a blood pressure of $110 / 74 \mathrm{mmHg}$, a pulse of 84 beats per minute, and a respiratory rate of 16 breaths per minute. He did not have any respiratory distress and saturation was maintained without oxygen support. Pulsation was felt on both carotid arteries, and his hemoglobin level on arrival was $9.3 \mathrm{~g} / \mathrm{dL}$.

Since the patient's bleeding had stopped, no further dressings or irrigation was done in the emergency ward, as doing so could have provoked life-threatening bleeding or caused aspiration. After arranging bloods for transfusion, he was taken to emergency surgery.

During surgery, the first step was to intubate the patient directly through the open wound. After intubation, the wound was examined thoroughly, followed by debridement and copious irrigation. Next, the airway was secured via tracheostomy and the endotracheal tubes were removed.

The cut was at the level of the hyoid bone, which was completely severed from the suprahyoid and the muscles of the floor of the mouth. The middle pharyngeal constrictor muscles were partially severed, and the epiglottis

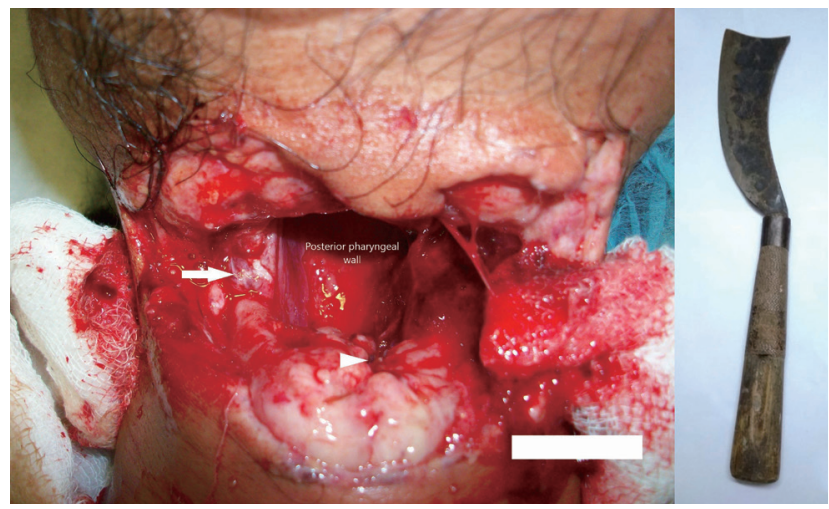

Fig. 1. At presentation to emergency department: wound crudely covered with gauze pieces, post pharyngeal wall noted through the wound, partly severed sternocleidomastoid muscles (white arrow), laryngeal inlet (white arrow head). with its fat pad was partly cut in the center. There were partial cuts on both the sternocleidomastoid muscles, but apart from the transected anterior jugular veins there was no other major vascular injury. The posterior hypopharynx wall could be seen through the open wound, but the vocal cords were spared because of their lower position (Fig. 2). After debridement, multiple holding sutures were made with catgut in order to realign the tissues and to evaluate the anatomy more precisely. Since the tissues were inflamed and covered in slough, no attempt was made to identify the muscles individually.

First, the partially cut epiglottis was repaired with interrupted Prolene 3-0 sutures. Next, the pharyngeal wall (middle pharyngeal constrictor muscles) and the base of the tongue were reconstructed with interrupted Vicryl 3-0 sutures in single layers, starting from the lateral aspect, taking care to oppose the mucosa. Then, the digastric muscle, stylohyoid, mylohyoid, omohyoid, geniohyoid, genioglossus, and hyoglossus muscles were sutured en masse, together with the strap muscles, and a few Vicryl sutures were hitched with the hyoid bone to maintain stability. A few loose Vicryl sutures were made over the partially cut sternocleidomastoid muscles. Irrigation was repeated, two drains were placed, and the skin was closed with interrupted nylon sutures. Since we expected

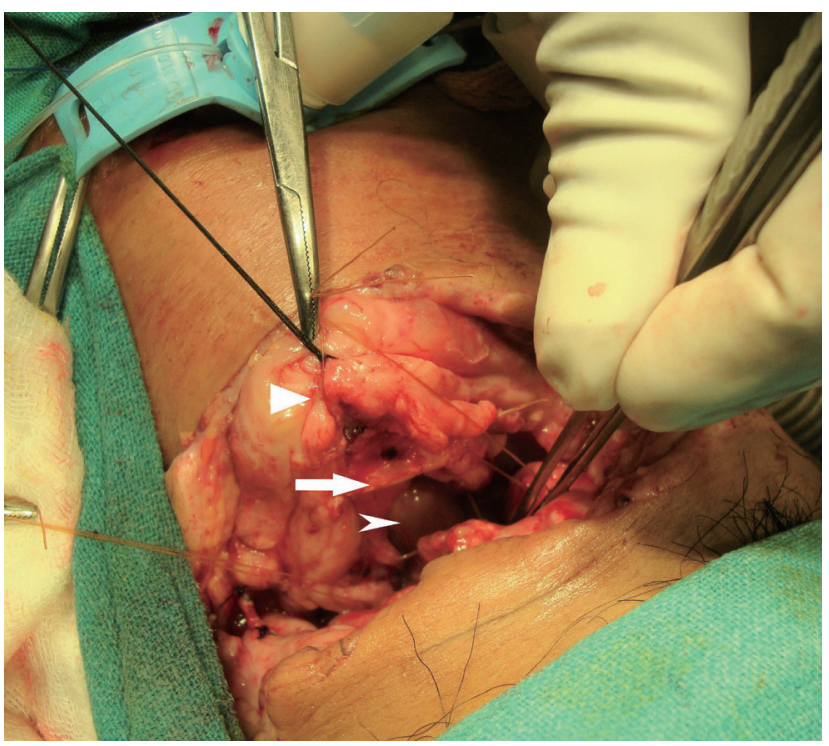

Fig. 2. Picture of the wound after tracheostomy has been secured. Strap muscles (triangular head), hyoid bone (arrow), posterior pharyngeal wall (arrow head). 
a prolonged recovery with potential wound infection, a pharyngocutaneous fistula, and problems with the patient's coughing, swallowing, and speech, a feeding gastrostomy was done.

The patient was observed in the intensive care unit for a day, feeding via gastrostomy was started on the second day, and sutures were removed on the seventh day. We gave him sips of water on the ninth day, and except for some mild coughing, he had no problem with swallowing and he could increase his liquid intake on the 10th day. A thin barium swallow was done on the 11th day, and likewise did not show any leaks or extravasation of dye (Fig. 3). On the 12th day, we performed a flexible laryngoscopy; except for some edema, the wound had healed well and both vocal cords were mobile. Based on these encouraging observations, we transitioned the patient to oral feeding with a soft diet and removed the tracheostomy on the 14th day. After tracheostomy removal, he could speak normally. The gastrostomy tube was kept and removed at the next outpatient department visit. The patient and his family members were counseled regularly, and at a 6-month follow-up visit, he was doing well and free from alcoholism.

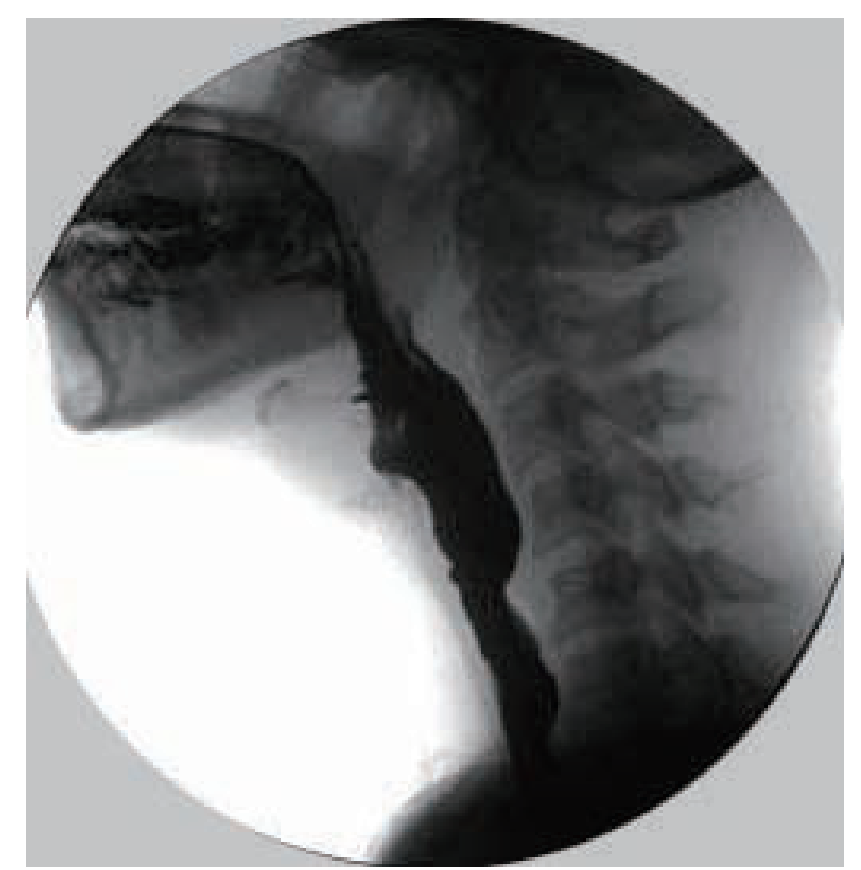

Fig. 3. Thin barium swallow: no spillage or extravasation of the dye can be seen.

\section{DISCUSSION}

Self-inflicted cut throat injuries with suicidal intent are rare in the Indian population [1]. To the best of our knowledge, this is the second report of successful management of a major suicidal cut throat injury [2]. Suicidal cut throat injuries are usually accompanied by hesitation cuts, and reports of cases without hesitation cuts are rare in the literature; in our patient, the absence of hesitation cuts showed his determination to commit the act [3]. He was fortunate that despite his horrific injury, his major vessels were spared, he did not experience any aspiration, and he did not develop any wound infection, even though proper care was delayed. The level of his injury corresponds to zone 2 of Roon and Christensen's classification of neck injuries, which is the most commonly injured zone [4,5].

Ryles tube management is the usual standard of care at many centers, but we performed a feeding gastrostomy because we were expecting a prolonged recovery and wound complications [6]. For securing the airway, a tracheostomy is the usual standard of management $[5,6]$.

Since there is no standardized oral intake protocol for patients with major cut throat injuries, we had to adapt from our experiences with laryngectomy patients. The usual protocol at Tata Medical Center for laryngectomy patients is, to start with spoonfuls of water as oral sips around the seventh day. If there is no aspiration and the patient is comfortable, oral intake is increased gradually. Barium swallows are not regularly done in all post-laryngectomy patients; instead, they are limited to only a few selected cases. The traditional method of carrying out a barium swallow before oral feeding has been challenged by several reports, White et al. [7], in a study of 259 laryngectomy patients, demonstrated a sensitivity of $26 \%$ and a specificity of $94 \%$ for barium swallows to predict pharyngocutaneous fistulas. Seven et al. [8] conducted a randomized controlled trial and concluded that early oral feeding on the first postoperative day is a safe practice with no relationship to fistula formation. Thus, it is safe to start oral feeding without doing a barium swallow test. However, since this patient presented late with a dirty wound, we delayed his oral intake relative to our usual practice.

Alcoholism is an established risk factor for suicide in 
India, and when compounded with family problems, suicidal ideation can be amplified $[9,10]$. These patients require regular counseling from trained counselors; as shown by Gøtzsche and Gøtzsche [11], behavioral therapies halve the risk for a repeated suicide attempt. Treatment is considered complete when the patient and family members are free from depression and helplessness and are involved in productive work.

Suicide was attempted using a dao (Naga sword), which is a heavy broad sword that varies from $45 \mathrm{~cm}$ to $65 \mathrm{~cm}$ in length and is mainly used in the northeastern part of India. Until roughly a century ago, the tradition of head-hunting was prevalent among the Nagas; consequently, the dao was fashioned over the years to be able to slice off the head or limbs with a single swing. Managing cut throat injuries is challenging and frightening even for experienced surgeons, but they are not beyond the scope of treatment. By maintaining proper surgical principles, many cases can be salvaged with acceptable outcomes.

\section{REFERENCES}

1. Radhakrishnan R, Andrade C. Suicide: an Indian perspective. Indian J Psychiatry 2012;54:304-19.

2. Naqvi Sayyed EH, Sadik A, Beg MH, Azam H, Nadeem R, Eram A. Successful management of suicidal cut throat injury with internal jugular, tracheal and esophageal transection: a case report. Trauma Case Rep 2017;13:30-4.

3. Shetty BSK, Padubidri JR, Bhandarkar AM, Shetty AJ, Shetty M. "Atypical suicidal" cut throat injury--a case report. J Forensic Leg Med 2009;16:492-3.

4. Alao T, Waseem M. Neck trauma [Internet]. Treasure Island (FL): StatPearls Publishing 2019 [cited 2019 Nov 7]. Available from: http://www.ncbi.nlm.nih.gov/books/NBK470422/.

5. Gilyoma JM, Hauli KA, Chalya PL. Cut throat injuries at a university teaching hospital in northwestern Tanzania: a review of 98 cases. BMC Emerg Med 2014;14:1.

6. Chakraborty D, Das C, Verma AK, Hansda R. Cut throat injury: our experience in rural set-up. Indian J Otolaryngol Head Neck Surg 2017;69:35-41.

7. White HN, Golden B, Sweeny L, Carroll WR, Magnuson JS, Rosenthal EL. Assessment and incidence of salivary leak following laryngectomy. Laryngoscope 2012;122:1796-9.

8. Seven H, Calis AB, Turgut S. A randomized controlled trial of early oral feeding in laryngectomized patients. Laryngoscope 2003;113:1076-9.

9. Gururaj G, Isaac MK, Subbakrishna DK, Ranjani R. Risk factors for completed suicides: a case-control study from Bangalore, India. Inj Control Saf Promot 2004;11:183-91.

10. Aggarwal S. Suicide in India. Br Med Bull 2015;114:127-34.

11. Gøtzsche PC, Gøtzsche PK. Cognitive behavioural therapy halves the risk of repeated suicide attempts: systematic review. J R Soc Med 2017;110:404-10. 